\title{
A Generalization of the Alexander Duality Theorem
}

\author{
W. S. MASSEY
}

§1. Introduction. According to the Alexander Duality Theorem, if $A$ is a closed subset of the $n$-sphere, $S^{n}$, the homology groups of the complement $S^{n}-A$, are independent of the manner in which $A$ is imbedded in $S^{n}$. This statement is still true if $S^{n}$ is replaced by a manifold which is a homology sphere; however, simple examples show that it is false if $S^{n}$ is replaced by an arbitrary manifold. It is the purpose of this paper to state and prove some theorems of this general nature which are true for imbeddings in arbitrary manifolds.

§2. Statement of results and examples. Let $X$ be a topological space and $G$ an abelian group. We will use the following notation for the various homology and cohomology groups which occur in the statements of our theorems:

$H_{q}(X ; G)=q$-dimensional singular homology group.

$H^{q}(X ; G)=q$-dimensional singular cohomology group.

$H_{c}^{q}(X ; G)=q$-dimensional Čech-Alexander-Spanier cohomology group of $X$ with compact supports.

$H_{q}^{\infty}(X ; G)=q$-dimensional Steenrod or Borel-Moore homology group of $X$.

For an easy method of defining $H_{q}^{\infty}(X ; G)$ and proving its principal properties, see Chapter 4 of [5]. We will only consider $H_{c}^{q}(X ; G)$ and $H_{q}^{\infty}(X ; G)$ for the case where $X$ is a locally compact Hausdorff space. The homology groups $H_{q}^{\infty}(X ; G)$ bear the same relation to the cohomology groups $H_{c}^{q}(X ; G)$ that the singular cohomology groups bear to the singular homology groups. If $X$ is compact and has the homotopy type of a finite CW-complex, then $H_{q}^{\infty}(X ; G) \approx$ $H_{q}(X ; G)$. If $X$ is a locally finite, finite dimensional $\mathrm{CW}$-complex, then $H_{q}^{\infty}(X ; G)$ may be computed by using infinite chains, i.e. chains which have an infinite number of non-zero coefficients (this fact motivates our notation for this homology theory).

Theorem I. Let $M$ be an orientable n-manifold, and let $h, k: A \rightarrow M$ be two imbeddings of $A$ as a closed subset of $M$. Then any one of the following four conditions suffices for the existence of isomorphisms 


$$
H_{q}(M-h(A) ; Z) \approx H_{q}(M-k(A) ; Z)
$$

for all $q$.

Condition 1: The maps $h$ and $k$ are properly homotopic.

Condition 2: The induced homorphisms $h_{\star}$ and $k_{\star}: H_{q}^{\infty}(A ; G) \rightarrow H_{q}^{\infty}(M ; G)$ are the same for all abelian groups $G$ and all $q$.

Condition 3: $H_{c}^{q}(A ; Z)$ is finitely generated for all $q$ and the induced homomorphisms $h_{\star}$ and $k_{\star}: H_{q}^{\infty}(A ; G) \rightarrow H_{q}^{\infty}(M ; G)$ are the same for all cyclic groups $G$ and all $q$.

Condition 4: $H_{c}^{q}(M ; Z)$ is finitely generated for all $q$ and the induced homomorphisms $h^{*}$ and $k^{*}: H_{c}^{q}(M ; G) \rightarrow H_{c}^{q}(A ; G)$ are the same for all cyclic groups $G$ and all $q$.

Remarks. (1) It should be emphasized that no conditions other than orientability are assumed on the manifold $M$ in Theorems I and II. In particular, $M$ need not be connected, compact, paracompact, triangulable, or differentiable.

(2) Regarding Condition 1, two maps $f_{0}, f_{1}: X \rightarrow Y$ are defined to be properly homotopic if there exists a homotopy $f: X \times I \rightarrow Y$ between them such that $f$ is a proper map, i.e., for any compact set $C \subset Y, f^{-1}(C)$ is compact.

(3) To say that $h: A \rightarrow M$ is an imbedding means that $h$ is a homeomorphism of $A$ onto a subset of $M$.

(4) It follows from the conclusion of Theorem I that the singular homology and cohomology groups with any coefficients of $M-h(A)$ and $M-k(A)$ are isomorphic (use the universal coefficient theorems).

We will now state Theorem II, which is dual to Theorem I in some sense.

Theorem II. Let $M$ be an orientable n-manifold, and let $h, k: U \rightarrow M$ be two imbeddings of $U$ as an open subset of $M$. Then any one of the following four conditions suffices for the existence of isomorphisms

$$
H_{c}^{q}(M-h(U) ; Z) \approx H_{c}^{q}(M-k(U) ; Z)
$$

for all $q$.

Condition 1: The maps $h$ and $k$ are homotopic.

Condition 2: The induced homomorphisms $h^{\star}$ and $k^{\star}: H^{q}(M ; G) \rightarrow$ $H^{q}(U ; G)$ are the same for all abelian groups $G$ and all $q$.

Condition 3: $H_{q}(M ; Z)$ is finitely generated for all $q$ and the induced homomorphisms $h^{\star}$ and $k^{\star}: H^{q}(M ; G) \rightarrow H^{q}(U ; G)$ are the same for all cyclic groups $G$ and all $q$.

Condition 4: $H^{q}(U ; Z)$ is finitely generated for all $q$ and the induced homomorphisms $h_{\star}$ and $k_{\star}: H_{q}(U ; G) \rightarrow H_{q}(M ; G)$ are the same for all cyclic groups $G$ and all $q$.

Remarks. (1) It follows from Brouwer's theorem on invariance of domain that if $h$ is an imbedding of $U$ as an open subset of $M$, then any other 
imbedding of $U$ in $M$ must also be as an open subset.

(2) Note that in Condition 1 we do not require that $h$ and $k$ be properly homotopic.

(3) The conclusion of Theorem II implies that for any $q$ and any abelian group $G$,

$$
\begin{aligned}
& H_{c}^{q}(M-h(U) ; G) \approx H_{c}^{q}(M-k(U) ; G), \\
& H_{c}^{\infty}(M-h(U) ; G) \approx H_{q}^{\infty}(M-k(U) ; G) .
\end{aligned}
$$

For the proof, see Chapter 4 of [5], especially Corollaries 4.18 and 4.25.

Next, we will consider a couple of simple illustrative examples.

Example 1. Let $M=S^{1} \times R$ (a cylinder) and $A=R$, the real line. Parameterize the circle $S^{1}$ by an angle $\theta$, where $-\pi \leq \theta \leq+\pi$. There are two topologically distinct ways to imbed $R$ in $S^{1} \times R$ as a closed set: $h: R \rightarrow$ $S^{1} \times R$ defined by $h(t)=\left(\theta_{0}, t\right)$ for any $t \in R$, and $k: R \rightarrow S^{1} \times R$ defined by

$$
k(t)= \begin{cases}\left(-\frac{\pi}{2},-t\right) & \text { for } t \leq-\frac{\pi}{2} \\ \left(t, \frac{\pi}{2}\right) & \text { for }-\frac{\pi}{2} \leq t \leq \frac{\pi}{2} \\ \left(\frac{\pi}{2}, t\right) & \text { for } \frac{\pi}{2} \leq t .\end{cases}
$$

These two imbeddings can be distinguished by the induced homomorphisms

$$
h_{\star}, k_{\star}: H_{1}^{\infty}(A ; Z) \rightarrow H_{1}^{\infty}(M ; Z) .
$$

Since $h_{\star} \neq k_{\star}$, Theorem $\mathrm{I}$ is not applicable; indeed, one can easily see that $H_{0}(M-h(A) ; Z) \neq H_{0}(M-k(A) ; Z)$, since $M-k(A)$ is connected, while $M-k(A)$ has two components. On the other hand, since $A$ is contractible, one can not distinguish between the two imbeddings $h$ and $k$ using singular theory. This example shows that it is necessary to use the Steenrod homology theory in the hypothesis of Theorem I.

One can modify this example so that Theorem II is applicable, as follows. Let $U=R \times(-\varepsilon, \varepsilon)$, where $\varepsilon$ is a small positive number and $(-\varepsilon, \varepsilon)$ denotes the open inteval $-\varepsilon<t<\varepsilon$. Let $h^{\prime}, k^{\prime}: U \rightarrow R \times S^{1}$ be imbeddings such that

$$
h^{\prime}(t, 0)=h(t), \quad k^{\prime}(t, 0)=k(t)
$$

for any $t \in R$, and $U$ is imbedded as a tubular neighborhood of $h(R)$ or $k(R)$ respectively. Then Theorem II is obviously applicable to prove that

$$
H_{c}^{q}\left(M-h^{\prime}(U) ; Z\right) \approx H_{c}^{q}\left(M-k^{\prime}(U) ; Z\right) .
$$


Example 2. Let $M$ be a compact orientable 2-manifold of genus 2, and let $A=S^{1}$. Let $h$ be a homotopically trivial imbedding of $S^{1}$ in $M$, and let $k$ be an imbedding which is homotopically non-trivial, but homologically trivial; such imbeddings are easy to find. In this example it does not matter whether one uses Steenrod homology theory or singular homology theory, since $A$ and $M$ are compact manifolds. Theorem $\mathrm{I}$ is applicable to show that

$$
H_{q}(M-h(A)) \approx H_{q}(M-k(A))
$$

for all $q$.

It would be easy to give more complicated examples to illustrate this theorem.

§3. Preparation for the proof. It is well known nowadays that the singular groups $H_{q}(X ; G)$ and $H^{q}(X ; G)$ may be defined from a free chain complex $C(X)$, by the formulas

$$
\begin{aligned}
& H_{q}(X ; G)=H_{q}(C(X) \otimes G), \\
& H^{q}(X ; G)=H^{q}(\operatorname{Hom}[C(X), G]) .
\end{aligned}
$$

Moreover, $C(X)$ is a covariant functor from the category of topological spaces and continuous maps to the category of chain complexes and chain maps.

Unfortunately, it is not so well known that there is a similar method of defining the groups $H_{c}^{q}(X ; G)$ and $H_{q}^{\infty}(X ; G)$. For any locally compact Hausdorff space $X$, there is defined a free cochain complex, $C_{c}^{*}(X)$, such that

$$
\begin{aligned}
& H_{c}^{q}(X ; G)=H^{q}\left(C_{c}^{*}(X) \otimes G\right), \\
& H_{q}^{\infty}(X ; G)=H_{q}\left(\operatorname{Hom}\left[C_{c}^{\star}(X), G\right]\right) .
\end{aligned}
$$

Moreover, $C_{c}^{*}(X)$, is a contravariant functor from the category of locally compact Hausdorff spaces and proper continuous maps to the category of cochain complexes and cochain maps. $C_{c}^{*}(X)$ is the Alexander-Spanier cochain complex of $X$ defined using finitely valued integral cochains with compact support. For more details on this subject, see [5], especially Chapters 1 and 4.

It is probably appropriate to review briefly the method of constructing the exact sequence of a pair in this homology and cohomology theory, since it is not a matter of common knowledge. Let $X$ be a locally compact Hausdorff space, $A$ a closed subset of $X$, and $U=X-A$ the open complement. We then have the following commutative diagram of cochain complexes and cochain maps:

$$
\begin{aligned}
& 0 \rightarrow C_{c}^{*}(X, A) \rightarrow C_{c}^{*}(X) \stackrel{i *}{\rightarrow} \quad C_{c}^{*}(A) \cdot \rightarrow 0 \\
& \uparrow \quad \| \\
& 0 \rightarrow C_{c}^{*}(U) \stackrel{\sigma}{\rightarrow} C_{c}^{*}(X) \rightarrow C_{c}^{*}(X) / C_{c}^{*}(U) \rightarrow 0 \text {. }
\end{aligned}
$$


Both rows of this diagram are short exact sequences. The vertical arrows induce isomorphisms on cohomology groups. Moreover, since all these cochain complexes consist of free abelian groups, the rows will remain exact if one applies the functor $\otimes G$ or $\operatorname{Hom}(, G)$. Thus we obtain the cohomology exact sequence of the pair $(X, A)$ :

$$
\ldots \stackrel{\delta^{*}}{\rightarrow} H_{c}^{q}(U ; G) \stackrel{\tau}{\rightarrow} H_{c}^{q}(X ; G) \stackrel{i^{*}}{\rightarrow} H_{c}^{q}(A ; G) \stackrel{\delta}{\rightarrow} H_{c}^{q+1}(U ; G) .
$$

For more details, see [5, Chapters 1 and 4].

Now suppose that $M$ is an arbitrary oriented $n$-dimensional manifold, $A$ is a closed subset of $M$, and $U=M-A$ is the open complement. Our proof of Theorems I and II will be based on the following commutative diagram (the coefficients group $G$ is systematically omitted from the notation):

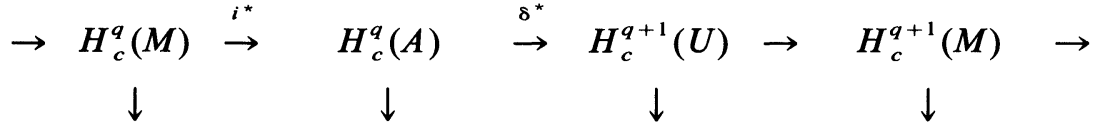

$$
\begin{aligned}
& \rightarrow H_{n-q}(M) \rightarrow H_{n-q}(M, U) \stackrel{\partial_{\star}}{\rightarrow} H_{n-q-1}(U) \stackrel{j_{\star}}{\rightarrow} H_{n-q-1}(M) \rightarrow \text {. }
\end{aligned}
$$

We will refer to this diagram as the "Main Diagram." The top line in this diagram is the cohomology exact sequence of the pair $(M, A)$, while the bottom line is the homology exact sequence of the pair $(M, U)$. The vertical arrows are Poincare duality isomorphisms. This diagram may be found in [1, expose 20], [5, page 357], or in various books on sheaf theory. In this diagram $i: A \rightarrow M$ and $j: U \rightarrow M$ denote inclusion maps.

The strategy of proof of Theorem I may now be described as follows: Suppose we have two imbeddings $h, k: A \rightarrow M$. Corresponding to each imbedding, there will be a main diagram of the type considered in the preceding paragraph. Our aim will be to impose conditions on the maps $h$ and $k$ which will insure that the cohomology exact sequences of the pairs $(M, h(A))$ and $(M, k(A))$ are isomorphic. It then follows from the isomorphisms in the main diagram that the homology exact sequences of the pairs $(M, M-h(A))$ and $(M, M-k(A))$ are isomorphic. In particular, the homology groups of $M-h(A)$ are isomorphic to those of $M-k(A)$, as desired.

The proof of Theorem II is similar. It uses the same diagram and general technique.

To carry out this program, we need some general propositions about chain complexes, especially free chain complexes.

§4. Some propositions about chain complexes. Completion of the proof. We will use standard notation and terminology for chain complexes, chain maps, chain homotopies, etc., see Dold [2, Chapter II], MacLane [3, §II. 2], Massey [6, $\S$ V. 2], or Spanier [8, Chapter 4, Section 1] for example. If $K$ and $K^{\prime}$ are chain complexes, and $f: K \rightarrow K^{\prime}$ is a chain map, we will use the abbreviations " $\operatorname{ker}(f)$ "' and "cok $(f)$ "' to denote the kernel and cokernel of $f$ respectively. In the case at hand, $\operatorname{ker}(f)$ is a subcomplex of $K$, and $\operatorname{cok}(f)$ 
is a quotient complex of $K^{\prime}$. We use this notation to state the following two propositions:

Proposition I. Let $f$ and $g: K \rightarrow K^{\prime}$ be chain maps, both of which are epimorphisms. Then any chain homotopy $D$ from $f$ to $g$ induces isomorphisms

$$
D_{\star}: H_{q}(\operatorname{ker}(f)) \rightarrow H_{q}(\operatorname{ker}(g))
$$

(for all $q$ ) such that the following diagram is commutative:

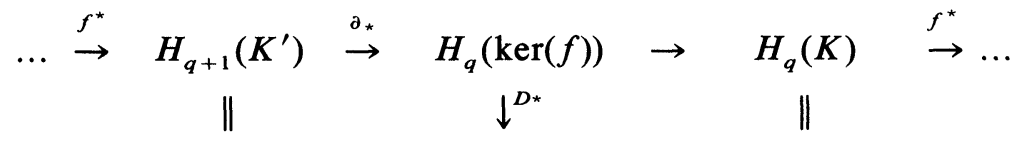

$$
\begin{aligned}
& \ldots \stackrel{g^{\star}}{\rightarrow} H_{q+1}\left(K^{\prime}\right) \stackrel{\partial_{\star}}{\rightarrow} \quad H_{q}(\operatorname{ker}(g)) \quad \rightarrow \quad H_{q}(K) \quad \stackrel{g^{\star}}{\rightarrow} \ldots
\end{aligned}
$$

In this diagram, the two horizontal lines are the homology exact sequences of the obvious short exact sequences of chain complexes.

Our next proposition is in some sense "dual" to the one just stated.

Proposition II. Let $f$ and $g: K \rightarrow K^{\prime}$ be the chain maps which are monomorphisms. Then any chain homotopy $D$ from $f$ to $g$ induces isomorphisms

$$
D_{\star}: H_{q}(\operatorname{cok}(f)) \rightarrow H_{q}(\operatorname{cok}(g))
$$

(for all $q$ ) such that the following diagram is commutative:

$$
\begin{aligned}
& \begin{array}{cccccc}
\stackrel{f_{\star}}{\rightarrow} & H_{q}\left(K^{\prime}\right) \\
\| & & \downarrow_{q}(\operatorname{cok}(f)) & \stackrel{\partial_{\star}}{\rightarrow} & H_{q-1}(K) & \stackrel{f_{\star}}{\rightarrow} \ldots
\end{array} \\
& \ldots \stackrel{g_{\star}}{\rightarrow} H_{q}\left(K^{\prime}\right) \quad \rightarrow \quad H_{q}(\operatorname{cok}(g)) \stackrel{\partial_{\star}}{\rightarrow} \quad H_{q-1}(K) \stackrel{g_{\star}}{\rightarrow} \ldots
\end{aligned}
$$

It would be nice if there were a brief direct definition of the isomorphism $D_{\star}$ in these two propositions, but unfortunately this does not seem possible. We will define them by means of the algebraic mapping cones of $f$ and $g$. We recall the definition: The algebraic mapping cone of the chain map $f: K \rightarrow K^{\prime}$ is the chain complex $M(f)=\left\{M(f)_{n}, d_{n}\right\}$ defined as follows:

$$
\begin{gathered}
M(f)_{n}=K_{n} \oplus K_{n+1}^{\prime} \quad \text { (direct sum). } \\
d_{n}\left(x, x^{\prime}\right)=\left(-\partial_{n}(x), \partial_{n+1}^{\prime}\left(x^{\prime}\right)+f_{n}(x)\right) \text { for any } x \in K_{n} \text { and } x^{\prime} \in K_{n+1}^{\prime} .
\end{gathered}
$$

Information about the algebraic mapping cone is available in many books, e.g., Dold [2, pages 18 and 25], MacLane [3, pages 46-47], Massey [5, pages $96-97$ and $360-361]$ or [6, pages $109-110]$, or Spanier [8, pages 166-167 and 191-192].

The obvious injection $i: K^{\prime} \rightarrow M(f)$ and projection $j: M(f) \rightarrow K$ are chain maps, and we have the following short exact sequence of chain complexes:

$$
0 \rightarrow K^{\prime} \stackrel{i}{\rightarrow} M(f) \stackrel{j}{\rightarrow} K \rightarrow 0 \text {. }
$$


On passing to homology, we obtain the following long exact sequence

$$
\rightarrow H_{q}\left(K^{\prime}\right) \stackrel{i_{\star}}{\rightarrow} H_{q-1}(M(f)) \stackrel{j_{\star}}{\rightarrow} H_{q-1}(K) \stackrel{d_{\star}}{\rightarrow} H_{q-1}\left(K^{\prime}\right) \rightarrow
$$

which is called "the homology exact sequence of the chain map $f$ ". In this exact sequence, the connecting homomorphism $d_{\star}$ is easily seen to be the same as the homomorphism $f_{*}: H_{q-1}(K) \rightarrow H_{q-1}\left(K^{\prime}\right)$ induced by $f$. Indeed, this is the main purpose of introducing the algebraic mapping cone, to imbed the induced homomorphisms $f_{*}$ in a long exact sequence in a natural way.

One of the main properties of the algebraic mapping cone of $f$ and the exact sequence of $f$ is their invariance under a chain homotopy of $f$. This may be stated as follows: Let $f$ and $g: K \rightarrow K^{\prime}$ be chain maps, and let $D$ be a chain homotopy from $f$ to $g$. Then $D$ induces a chain map $D^{\prime}: M(f) \rightarrow M(g)$ by the formula $D^{\prime}\left(x, x^{\prime}\right)=\left(x, x^{\prime}+D(x)\right)$. Moreover, the following diagram of chain complexes and chain maps is commutative:

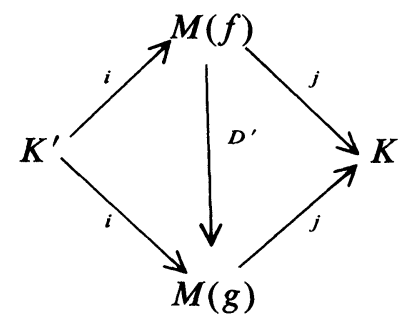

By writing down the homology exact sequences of $f$ and $g$ and using the 5-lemma, we see that the induced homomorphism $D_{\star}^{\prime}: H_{q}(M(f)) \rightarrow H_{q}(M(q))$ is an isomorphism. Moreover, the homology sequences of $f$ and $g$ are isomorphic.

Next, assume that $f: K \rightarrow K^{\prime}$ is a chain map which in addition is an epimorphism. Then there is a natural chain map

$$
\alpha: \operatorname{ker}(f) \rightarrow M(f)
$$

defined by $\alpha(x)=(x, 0)$. It is readily verified that the following diagram (which involves two exact sequences) is commutative:

$$
\ldots \stackrel{f_{\star}}{\rightarrow} H_{q+1}\left(K^{\prime}\right) \overbrace{i_{\star}}
$$

It follows from the 5-lemma that $\alpha_{\star}$ is an isomorphism.

We will now consider the dual situation. Let $f: K \rightarrow K^{\prime}$ be a chain map which is a monomorphism. In this case there is a natural chain map

$$
\beta: M(f) \rightarrow \operatorname{cok}(f)
$$


defined by $\beta\left(x, x^{\prime}\right)=\eta\left(x^{\prime}\right)$; where $\eta: K^{\prime} \rightarrow \operatorname{cok}(f)$ is the natural projection. It can now be checked that the following diagram is commutative:

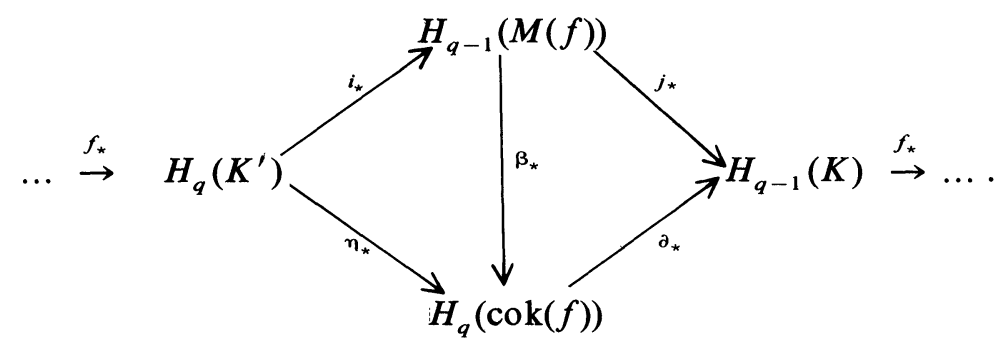

Once again the 5-lemma implies that $\beta_{*}$ is an isomorphism.

It is now an easy matter to prove Proposition I. The isomorphism $D_{*}: H_{q}(\operatorname{ker}(f)) \rightarrow H_{q}(\operatorname{ker}(g))$ is defined to be the composition of the following three isomorphisms:

$$
H_{q}(\operatorname{ker}(f)) \stackrel{\alpha_{\star}}{\rightarrow} H_{q}(M(f)) \stackrel{D_{\star}^{\prime}}{\rightarrow} H_{q}(M(g)) \stackrel{\alpha_{\star}}{\leftarrow} H_{q}(\operatorname{ker}(g)) .
$$

Similarly, to prove Proposition II, we define the isomorphism $D_{\star}$ : $H_{q}(\operatorname{cok}(f)) \rightarrow H_{q}(\operatorname{cok}(g))$ to be the composition of the following three isomorphisms:

$$
H_{q}(\operatorname{cok}(f)) \stackrel{\beta_{\star}}{\leftarrow} H_{q-1}(M(f)) \stackrel{D_{\star}^{\prime}}{\rightarrow} H_{q-1}(M(g)) \stackrel{\beta_{\star}}{\rightarrow} H_{q}(\operatorname{cok}(g)) .
$$

The remaining details of the proofs of Propositions I and II are left to the reader.

Corollary of Proposition I. Let $M$ be an oriented n-manifold, and let $h$, $k: A \rightarrow M$ be imbeddings of $A$ as a closed subset of $M$. Assume that the induced cochain maps $h^{\#}, k^{\#}: C_{c}^{*}(M) \rightarrow C_{c}^{*}(A)$ are cochain homotopic. Then $H_{q}(M-h(A) ; Z) \approx H_{q}(M-k(A) ; Z)$ for all $q$.

Corollary of Proposition II. Let $M$ be an oriented n-manifold, and let $h, k: U \rightarrow M$ be imbeddings of $U$ as an open subset of $M$. Assume the induced chain maps $h_{\#}, k_{\#}: C(U) \rightarrow C(M)$ are chain homotopic. Then $H_{c}^{q}(M-h(U) ; Z) \approx H_{c}^{q}(M-k(U) ; Z)$ for all $q$.

These corollaries follow from the corresponding propositions by use of the Main Diagram of $\S 3$.

In order to complete the proof of Theorems I and II we must develop sufficient conditions for two chain maps to be chain homotopic. In order to do this, we will need to assume that the chain complexes are free, i.e., that they are chain complexes of free abelian groups. So far in this section we have not needed this assumption.

Proposition III. Given any free chain complex $L=\left\{L_{n}, d_{n}\right\}$, there exists a sequence of cohomology classes $e_{q} \in H^{q}\left(\operatorname{Hom}\left[L, H_{q}(L)\right]\right)$ having the following property: For any free chain complex $K$ and chain maps $f, g: K \rightarrow L, f$ 
and $g$ are chain homotopic if and only if $f^{*}\left(e_{q}\right)=g^{*}\left(e_{q}\right)$ for all $q$ (note: We do not assert that the cohomology classes $e_{q}$ are unique).

Sketch of proof. Define a chain complex $C=\left\{C_{q}, \partial_{q}\right\}$ to be short if there exists an integer $n$ such that $C_{q}=0$ except for $q=n$ or $n+1$, and $\partial_{n+1}: C_{n+1} \rightarrow C_{n}$ is a monomorphism. It is well known (and easy to prove) that any free chain complex is a (locally finite) direct sum of short chain complexes.

First one proves the proposition for the special case where $L$ is a short chain complex, i.e., $L_{q}=0$ except for $q=n$ or $n+1$, and $d_{n+1}$ is a monomorphism. Let $e_{n}^{\prime}: L_{n} \rightarrow H_{n}(L)$ be the homomorphism which assigns to each element of $L_{n}$ its homology class. Then $e_{n}^{\prime}$ is an element of the cochain complex $\operatorname{Hom}\left(L, H_{n}(L)\right)$, and it is readily seen to be a cocycle. Let $e_{n}$ denote its cohomology class. It is now an easy exercise to prove that if $f^{\star}\left(e_{n}\right)=g^{*}\left(e_{n}\right)$, then $f$ is chain homotopic to $g$, as required.

The general case follows easily from this special case by making use of the properties of (locally finite) direct sums.

Corollary 1 of Proposition III. Let $K$ and $L$ be free chain complexes. Then two chain maps $f$ and $g: K \rightarrow L$ are chain homotopic if and only if the induced homomorphisms $f^{*}$ and $g^{*}: H^{q}(\operatorname{Hom}(L, G)) \rightarrow H^{q}(\operatorname{Hom}(K, G))$ are the same for all abelian groups $G$ and all integers $q$.

Corollary 2. Let $X$ and $Y$ be locally compact Hausdorff spaces, and let $f$ and $g: X \rightarrow Y$ be properly homotopic maps. Then the induced cochain maps $f^{\#}$ and $g^{\#}: C_{c}^{*}(Y) \rightarrow C_{c}^{*}(X)$ are cochain homotopic.

Proof. It is proved in Chapter 4 of [5] that the induced homomorphisms $f_{*}$ and $g_{*}: H_{q}^{\infty}(X ; G) \rightarrow H_{q}^{\infty}(Y ; G)$ are the same for all abelian groups $G$ and all $q$. One now applies Corollary 1 to reach the desired conclusion. Note the contrast with singular homology theory: In singular theory one proves that homotopic maps induce chain homotopic chain maps, and then one concludes that they induce the same homomorphism. In Alexander-Spanier theory as outlined in Chapters 1 and 4 of [5], one proves directly that properly homotopic maps induced the same homomorphism on cohomology and homology, and then one concludes that the induced cochain maps are cochain homotopic.

Corollary 3. Let $K$ and $L$ be free chain complexes, and assume that $H_{q}(L)$ is finitely generated for all $q$. Then two chain maps $f, g: K \rightarrow L$ are chain homotopic if and only if the induced homomorphisms $f^{*}$ and $g^{*}: H^{q}(\operatorname{Hom}(L, G)) \rightarrow H^{q}(\operatorname{Hom}(K, G))$ are the same for all cyclic groups $G$ and all $q$.

This follows readily from Proposition III, using the fact that every finitely generated abelian group is a direct sum of cyclic groups.

In view of Proposition III and its corollaries, it is natural to raise the following question: Suppose that $K$ and $L$ are free chain complexes and 
$f, g: K \rightarrow L$ are chain maps such that the induced homomorphisms $f_{\star}$ and $g_{\star}: H_{q}(K \otimes G) \rightarrow H_{q}(L \otimes G)$ are the same for all abelian groups $G$ and all $q$. Does it follow that $f$ and $g$ are chain homotopic?

The following example shows that the answer to this question is no. Choose free chain complexes $K$ and $L$ such that

$$
\begin{aligned}
& H_{q}(K)=\left\{\begin{array}{lll}
Q & \text { for } & q=n-1 \\
0 & \text { for } & q \neq n-1
\end{array}\right. \\
& H_{q}(L)=\left\{\begin{array}{lll}
Z & \text { for } & q=n \\
0 & \text { for } & q \neq n .
\end{array}\right.
\end{aligned}
$$

It is not difficult to prove that $\operatorname{Ext}(Q, Z)$ is an uncountable abelian group. From this it follows that there are uncountably many distinct chain homotopy classes of chain maps $f: K \rightarrow L$ (use Theorem 4.3 on page 78 of MacLane [3]).

By using the universal coefficient theorem for homology, one can show that for any abelian group $G$,

$$
\begin{aligned}
H_{q}(K \otimes G)=0 & \text { for } q \neq n-1, \\
H_{q}(L \otimes G)=0 & \text { for } q \neq n .
\end{aligned}
$$

Hence for any chain map $f: K \rightarrow L$, the induced homomorphism $f_{\star}: H_{q}(K \otimes G) \rightarrow H_{q}(L \otimes G)$ is zero for all $q$.

On the other hand, Corollary 3 does apply to this example; in fact, it is only necessary to choose $G$ an infinite cyclic group to distinguish between the various chain homotopy classes.

Proposition IV. Let $K$ be a free chain complex such that $H_{q}(K)$ is finitely generated for all $q$, and let $L$ be a torsion free chain complex. Then two chain maps $f, g: K \rightarrow L$ are chain homotopic if and only if $f_{\star}=$ $g_{*}: H_{q}(K \otimes G) \rightarrow H_{q}(L \otimes G)$ for all cyclic groups $G$ and all $q$.

Sketch of proof. The assumptions on $K$ imply that it is chain homotopy equivalent to a chain complex of finitely generated free abelian groups (cf. [6, Lemma 11.1, page 194]). Hence we may assume that $K$ is a chain complex of finitely generated abelian groups without changing the problem. This implies that $K$ is a direct sum of so-called "elementary" chain complexes (a chain complex is elementary if it is short, and each non-zero chain group is infinite cyclic); for a proof of this assertion, see Dold [2, page 27]. From this it follows that it suffices to prove the proposition in the special case where $K$ is an elementary chain complex. The proof for this case is quite easy; the details are left to the reader.

With these preparations taken care of, it is now an easy matter to prove Theorems I and II. If we apply Proposition IV and the various corollaries of Proposition III to the corollary of Proposition I, we obtain Theorem I. Similarly if we apply Proposition IV and the various corollaries of Proposition 
III to the corollary of Proposition II, we obtain Theorem II. The details are left to the reader.

§5. Final remarks. The crux of this paper is the proof that certain group extensions in the homology or cohomology exact sequence are invariant under chain homotopies. This technique has already been used in Malm [4, Section 3] to prove an old result of Arnold Shapiro about the cohomology of sphere bundles. This technique was also used in Massey [7]. Perhaps it has other applications.

An astute reader may have noticed that Conditions 2 and 3 in the statement of Theorem I can be weakened somewhat, in view of Proposition III. It is not necessary to consider all groups $G$ in Condition 2, or all cyclic groups $G$ in Condition 3. Similar remarks apply to Theorem II. Also, the conclusion of Theorem I can be strengthened to read as follows: The homology sequences of the pairs $(M, M-h(A))$ and $(M, M-k(A))$ are isomorphic if any one of the four conditions holds. The conclusion of Theorem II can be strengthened in a similar way.

\section{REFERENCES}

1. H. Cartan, Séminaire Henri Cartan, 3e annee: 1950/51. Cohomologie des Groupes, Suite Spectrale, Faisceaux.

2. A. Dold, Lectures on Algebraic Topology, Springer-Verlag, New York, 1972.

3. S. Maclane, Homology, Springer-Verlag, New York, 1963.

4. D. MALM, Concerning the cohomology ring of a sphere bundle, Pacific J. Math. 9 (1959), 1191-1214.

5. W. S. MAssey, Homology and Cohomology Theory: An Approach Based on Alexander-Spanier Cochains, Marcel Dekker, New York, 1978.

6. W. S. MASSEY, Singular Homology Theory, (Graduate texts in mathematics no. 70), SpringerVerlag, New York, 1980.

7. W. S. MASSEY, On the universal coefficient theorem of Eilenberg and MacLane, Bol. Soc. Math. Mex. 3 (1958), 1-12.

8. E. Spanier, Algebraic Topology, McGraw-Hill, New York, 1966.

The author acknowledges with gratitude National Science Foundation Grant MCS 78-02977.

Yale University, New Haven, CT 06520

Received March 4, 1980 